\title{
Confronting Models of Massive Star Evolution and Explosions with Remnant Mass Measurements
}

\author{
Carolyn A. Raithel ${ }^{1}$ (1) , Tuguldur Sukhbold ${ }^{2,3}$ (10), and Feryal Özel ${ }^{1,4}$ \\ ${ }^{1}$ Department of Astronomy and Steward Observatory, University of Arizona, 933 N. Cherry Avenue, Tucson, AZ 85721, USA \\ ${ }_{2}^{2}$ Department of Astronomy, The Ohio State University, Columbus, 140 W. 18th Avenue, Columbus, OH 43210, USA \\ ${ }^{3}$ Center for Cosmology and AstroParticle Physics, The Ohio State University, $191 \mathrm{~W}$. Woodruff Avenue, Columbus, OH 43210, USA \\ Received 2017 November 29; revised 2018 February 15; accepted 2018 February 15; published 2018 March 22
}

\begin{abstract}
The mass distribution of compact objects provides a fossil record that can be studied to uncover information on the late stages of massive star evolution, the supernova explosion mechanism, and the dense matter equation of state. Observations of neutron star masses indicate a bimodal Gaussian distribution, while the observed black hole mass distribution decays exponentially for stellar-mass black holes. We use these observed distributions to directly confront the predictions of stellar evolution models and the neutrino-driven supernova simulations of Sukhbold et al. We find strong agreement between the black hole and low-mass neutron star distributions created by these simulations and the observations. We show that a large fraction of the stellar envelope must be ejected, either during the formation of stellar-mass black holes or prior to the implosion through tidal stripping due to a binary companion, in order to reproduce the observed black hole mass distribution. We also determine the origins of the bimodal peaks of the neutron star mass distribution, finding that the low-mass peak (centered at $\sim 1.4 M_{\odot}$ ) originates from progenitors with $M_{\text {ZAMS }} \approx 9-18 M_{\odot}$. The simulations fail to reproduce the observed peak of highmass neutron stars (centered at $\sim 1.8 M_{\odot}$ ) and we explore several possible explanations. We argue that the close agreement between the observed and predicted black hole and low-mass neutron star mass distributions provides new, promising evidence that these stellar evolution and explosion models capture the majority of relevant stellar, nuclear, and explosion physics involved in the formation of compact objects.
\end{abstract}

Key words: stars: black holes - stars: evolution - stars: neutron - supernovae: general

\section{Introduction}

The masses of compact objects that are formed through massive star evolution are relics of the various physical processes that take place during the star's lifetime and subsequent death. First, whether the star explodes and forms a neutron star or implodes to form a black hole is largely determined by the advanced-stage evolution in the stellar core. Beyond the star's fate, the pre-supernova (pre-SN) core structure also influences the mass of the resulting neutron star following a successful explosion. If instead the star implodes, the black hole mass is affected by the star's mass loss history. Second, the key processes that take place during the core collapse itself, such as neutrino transport and multidimensional turbulence, can also influence the nature of the outcome. Furthermore, the dense matter equation of state plays a role in determining the possible range of neutron star and black hole masses, setting both the maximum neutron star mass and potentially the minimum black hole mass (Rhoades \& Ruffini 1974; Kalogera \& Baym 1996; Chamel et al. 2013; for the black hole case see, e.g., Zhang et al. 2008). Because the mass distribution of compact objects is collectively shaped by each of these processes, it has the potential to provide insight into the fundamental physics underlying stellar evolution, the $\mathrm{SN}$ explosion mechanism, and the dense matter equation of state.

Observationally, the mass distribution can be inferred from the known sample of neutron star and black hole masses. Many black hole masses have been measured from X-ray binary systems, while over 30 precision neutron star masses have been

\footnotetext{
${ }^{4}$ Guggenheim Fellow.
}

measured from double neutron stars and millisecond pulsars (for a recent review of the latter, see Özel \& Freire 2016). To infer the black hole mass distribution, Özel et al. (2010) combined measurements from 16 low-mass X-ray binaries, finding that it follows an exponential decline. Farr et al. (2011) fit a similar sample of black hole masses from 15 low-mass $\mathrm{X}$-ray binaries, but also included black holes from five highmass X-ray binaries. They found that the low-mass population follows a power-law distribution, while the combined population follows an exponential decline. The mass distribution of neutron stars has also been measured, with an ever-growing and precise sample (Thorsett \& Chakrabarty 1999; Özel et al. 2012; Kiziltan et al. 2013; Antoniadis et al. 2016). The most recent study by Antoniadis et al. (2016) inferred a bimodal distribution, possibly indicating two separate formation channels for creating neutron stars.

From the theoretical side, there have been recent new developments in our understanding of the evolution of massive stars and the modeling of their explosions. In particular, during the advanced stages of core evolution of massive stars, the interplay of convective burning episodes of carbon and oxygen gives rise to final pre-SN structures that are non-monotonic in initial mass (Sukhbold \& Woosley 2014). The pre-SN core structure of a massive star, i.e., the density gradient surrounding the iron core, is known to play a pivotal role in determining whether the star explodes or implodes (e.g., Burrows et al. 1995). Several recent studies have explored the connection between this final core structure and the landscape of neutrino-driven explosions of massive stars through numerical and semi-analytical approaches (O'Connor \& Ott 2011; Ugliano et al. 2012; Pejcha \& Thompson 2015; Ertl et al. 2016; Müller et al. 2016; Sukhbold et al. 2016; Murphy \& Dolence 2017). While these works differ in 
their scope and complexity, all find that there is no single initial mass below which stars only explode and above which only implode. Instead, they recover a much more complicated landscape of explosions as dictated by the pre-SN evolution of massive stars.

A number of previous studies have explored the connection between the physical processes of the $\mathrm{SN}$ and the observed distribution in compact objects. For example, Pejcha et al. (2012) used the mass distribution of double neutron stars to constrain the entropy coordinate in the progenitor at which the explosion must originate. Fryer et al. (2012) calculated the remnant mass distributions for different explosion mechanisms and metallicities, both for single and binary stars, to determine the relative effect of each factor. In another work, Belczynski et al. (2012) found that the existence of a gap between the most massive neutron stars and least massive black holes may depend soley on the growth timescales of the instabilities driving the explosion, while Kochanek (2014) showed that the observed gap could be naturally explained by the unbinding of the hydrogen envelope during failed $\mathrm{SNe}$, via the Nadyozhin-Lovegrove mechanism (Nadezhin 1980; Lovegrove \& Woosley 2013). These studies, however, all used mass cuts, rather than realistic explosion simulations, to determine the predicted remnant masses.

Sukhbold et al. (2016; hereafter S16) surveyed the explosion outcomes, including the nucleosynthesis yields, light curves, and compact remnants, for a large set of stellar models using a novel one-dimensional neutrino-driven explosion mechanism, based on Ugliano et al. (2012) and Ertl et al. (2016). While three-dimensional models are the gold standard in understanding SN explosions, they are computationally expensive and prohibit an exploration of a large parameter space. Furthermore, although great progress is being made in multidimensional explorations of the problem, the community has not yet reached a consensus on the explosion mechanism of the core-collapse SNe (Janka et al. 2016 and references therein). Though simplified, the one-dimensional treatment in S16 allows for large parameter-space studies. In that work, the authors explored the outcomes due to various parameterizations of the central engine applied to 200 pre-SN stars with initial masses between 9 and $120 M_{\odot}$. They performed a preliminary comparison of the remnant masses produced in their simulations to the observed populations of black holes and neutron stars, and found reasonable agreement in the produced mass range. However, the comparison was qualitative as they did not properly weight the observed masses or explore any observational biases.

This new, fine grid of stellar evolution models, combined with a simplified, parametric explosion mechanism and combined with a better quantitative understanding of remnant mass distributions, now make it possible to confront these theoretical models with remnant mass observations in a systematic way. One potential difficulty in such a comparison, however, is that the theoretical models describe single-star evolution, while all precision mass measurements come from binary systems. We argue that a meaningful comparison can nevertheless be made for the following reasons.

For late-time mass transfers (cases B and C; Smith 2014), the He-core mass, which is the main determinant for the final pre-SN structure (Sukhbold \& Woosley 2014), is not appreciably affected by the mass transfer. Thus, for these scenarios, the remnant outcome will not be strongly affected by binary evolution. On the other hand, in early stable mass transfers via Roche-lobe overflow, the He core mass can be affected. However, this effect can be at least partially encompassed by a stronger mass loss efficiency in the single-star models. In other words, even though the models here describe single-star evolution, due to the uncertain nature of mass loss (e.g., Renzo et al. 2017), the single-star models can be representative of some close binary scenarios. We revisit this point in Section 6.3. For millisecond pulsars, which are spun up by accretion from their binary companion after forming, Antoniadis et al. (2016) found that the accretion rates onto the neutron star are very inefficient, and that the observed masses must be close to their birth masses. Thus, we take the approach in this paper that comparing the remnant masses measured from binary systems to theoretical models of single-star evolution indeed can provide a reasonable first constraint on the theoretical models.

With these motivations, we directly confront the stellar evolution models and SN outcomes of S16 with the observed black hole and neutron star mass distributions. We describe the stellar evolution and SN models in more detail in Section 2. In Section 3, we review the current collection of observed compact object masses. In Section 4, we compute the simulated black hole mass distribution, in order to compare it to the observed distribution on a level playing field. We find that the fraction of the stellar envelope that must be ejected during the SN implosion in order to recreate the observed black hole mass distribution is quite large. In Section 5, we compute the mass distribution of neutron star remnants and find surprisingly close agreement between the simulated outcomes and low-mass peak of the observed bimodal distribution of Antoniadis et al. (2016). We explore the origin of this peak and find that it originates from progenitors with zero-age main sequence masses $\left(M_{\text {ZAMS }}\right)$ in the range $M_{\text {ZAMS }} \approx 9-18 M_{\odot}$. We discuss in Section 6 possible explanations for the lack of high-mass neutron stars and LIGO-mass black holes in the simulations. Finally, we discuss the possible implications of our inferred distributions for stellar evolution and explosion models more generally.

\section{Pre-SN Stellar Evolution and Explosion}

The nucleosynthesis yields, remnant masses, and light curves due to neutrino-driven explosions from a wide range of solar metallicity massive stars were surveyed recently in S16. In the following, we briefly highlight aspects of that work that are relevant to the present study.

All of the progenitor models used in S16 were computed using the one-dimensional implicit hydrodynamics code KEPLER (Weaver et al. 1978). The main progenitor set consists of 200 non-rotating, solar metallicity models with initial masses between 9 and $120 M_{\odot}$, and were mostly compiled from Woosley \& Heger (2007), Sukhbold \& Woosley (2014), and Woosley \& Heger (2015). Between the initial masses of 13 and $30 M_{\odot}$, the models were calculated with $0.1 M_{\odot}$ increments. As will be shown in Section 5 , the large number of models with fine resolution in initial mass space were critical in uncovering discrete branches in the neutron star mass distribution.

Mass loss rates from Nieuwenhuijzen \& de Jager (1990) were employed in all models. While the lightest stars do not lose much mass throughout their evolution, the mass loss gradually strengthens with increasing initial mass. The entire envelope was lost for stars with initial masses above $40 \mathrm{M}_{\odot}$ 
and a Wolf-Rayet wind from Wellstein \& Langer (1999) was adopted for these stars. Due to coarse sampling in mass space for high- $M_{\text {ZAMS }}$ stars and due to the assumed input physics, nearly all Wolf-Rayet pre-SN stars lost their He-envelopes as well, and therefore died as carbon-oxygen (CO) cores.

Although the He core mass, and hence the final pre-SN structure, is insensitive to mass loss for the lighter stars that do not lose all of their envelope, the masses of black holes that are formed if the star implodes have an appreciable dependence on the adopted prescription of mass loss. For stars with $M_{\text {ZAMS }}<40 M_{\odot}$, the amount of envelope remaining sets the range of possible black hole masses upon implosion, while for stars with $M_{\text {ZAMS }}>40 M_{\odot}$, the final star mass approximately sets the possible black hole mass.

The final fates of KEPLER pre-SN progenitors were modeled from the onset of core collapse through core bounce and to either a successful explosion or implosion with the Prometheus-Hot Bubble code ( $\mathrm{P}-\mathrm{HOTB}$, Janka \& Mueller 1996; Kifonidis et al. 2003). P-HOTB is a one-dimensional Eulerian hydrodynamics code with a simplified gray neutrinotransport solver and a high-density equation of state (Lattimer \& Swesty 1991, with $K=220 \mathrm{MeV}$ ). The simulations were run for sufficiently long times in order to determine the final mass cuts and explosion energies. For technical details and further discussion see Ugliano et al. (2012) and Ertl et al. (2016).

A major improvement of the recent studies such as S16, Ugliano et al. (2012), and Ertl et al. (2016) over the previous surveys is that the explosion outcomes were free from arbitrary mass cuts and directly dialed-in explosion energies (e.g., Woosley et al. 2002; Chieffi \& Limongi 2013; Nomoto et al. 2013). This was achieved by calibrating the free parameters of an analytic proto-neutron star (PNS) cooling model to reproduce the observables of SN1987A for five different models of the progenitor star: W15, W18, W20, N20, and $\mathrm{S} 19.8$, for progenitors more massive than $12 M_{\odot}$. Each model resulted in a particular choice of parameters (which we call the "central engine" henceforth) and each central engine was then applied to the 200 KEPLER pre-SN models. Furthermore, S16 improved the low ZAMS-mass end compared to Ugliano et al. (2012) by adding SN 1054 as a calibration anchor and interpolating the core parameters to account for the reduced PNS contraction in the case of small PNS masses. For these lighter progenitors $\left(M_{\text {ZAMS }}<12 M_{\odot}\right)$, the authors of S16 used the model Z9.6, for which all stars successfully explode. Finally, each explosion yielded a unique set of observational outcomes, including the remnant mass, that is characterized by the pre-SN core structure of the progenitor. This, in turn, allowed us to construct the expected compact remnant mass distributions for each central engine.

Figure 1 shows the baryonic remnant masses produced by one sample engine combination $(\mathrm{Z} 9.6+\mathrm{W} 18)$ as a function of the initial progenitor mass. A successful explosion results in a neutron star for most models (purple), but in a very small number of cases that experienced significant amount of fallback, a light black hole is formed (gray stars). Since such fallback black holes occur infrequently and only at relatively high-mass models, we omitted them from our analysis. ${ }^{5}$

\footnotetext{
5 We repeated the analyses described in Section 4 with an additional branch to model fallback black holes with a Gaussian distribution. This led to a negligible change in the results.
}

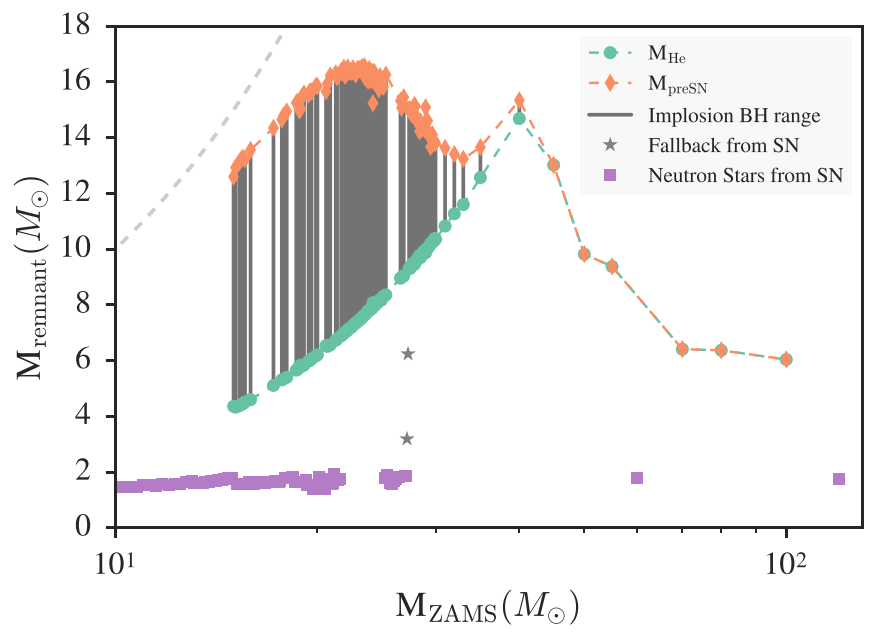

Figure 1. Baryonic remnant masses as a function of the progenitor ZAMS mass, for the central engines Z9.6+W18. Neutron star remnant masses from successful explosions are shown in purple. The range of possible black hole masses, shown in gray, is bound by the He-core mass (green circles) and pre$\mathrm{SN}$ mass (orange diamonds) of the progenitor, because an uncertain fraction of the stellar envelope may be ejected either during the formation of the black hole, via the Nadyozhin-Lovegrove mechanism (Nadezhin 1980; Lovegrove \& Woosley 2013), or prior to the implosion by tidal stripping from a binary companion. The gray dashed line denotes the initial progenitor mass. Note the co-existence of neutron star and black hole outcomes between $M_{\text {ZAMS }} \sim$ 15-21 $M_{\odot}$ and 25-28 $M_{\odot}$.

Although a failed explosion would certainly form a stellarmass black hole, its exact mass is not well determined for progenitors that retain some amount of envelope by the time of implosion (i.e., for stars with $M_{\text {ZAMS }}<40 M_{\odot}$ ). A weak shock, which is launched by the loss of the PNS binding energy in the Nadyozhin-Lovegrove effect, may be able to eject a fraction or all of the remaining envelope (Nadezhin 1980; Lovegrove \& Woosley 2013; Coughlin et al. 2017; Fernández et al. 2018). Additionally, it is possible that some of the progenitor envelope may be stripped by a binary companion prior to the implosion. Thus, we might expect that a fraction of all remnant black holes come from the collapse of stripped cores. The black hole masses from stellar implosions (gray lines in Figure 1) are thus bounded by the He-core (green circles) and the final preSN (orange diamonds) masses of the progenitor, and ultimately depend on how much of the stellar envelope gets ejected during or prior to the implosion. We analyze this further in Section 4.

Finally, we note the presence of large intervals in $M_{\text {ZAMS-space over which the outcomes switch between neutron }}$ stars and black holes in Figure 1. As has previously been reported, the explodability of the pre-SN star is not determined only by the initial mass (O'Connor \& Ott 2011; Ugliano et al. 2012; Pejcha \& Thompson 2015; Ertl et al. 2016; Müller et al. 2016; S16; Murphy \& Dolence 2017). For example, Ertl et al. (2016) propose a new two-dimensional parameter space to characterize pre-SN stars and to predict whether a neutron star or a black hole forms following core collapse. Specifically, a separatrix can be drawn in this space such that any model that falls above it will explode, while models below it will implode. In this framework, we interpret the large intervals of neutron star or black hole outcomes to result from repeated crossings of this separatrix as the initial mass varies and the core structure changes. In addition, there are smaller intervals in initial-mass space, such as between $M_{\text {ZAMS }} \sim 15$ 


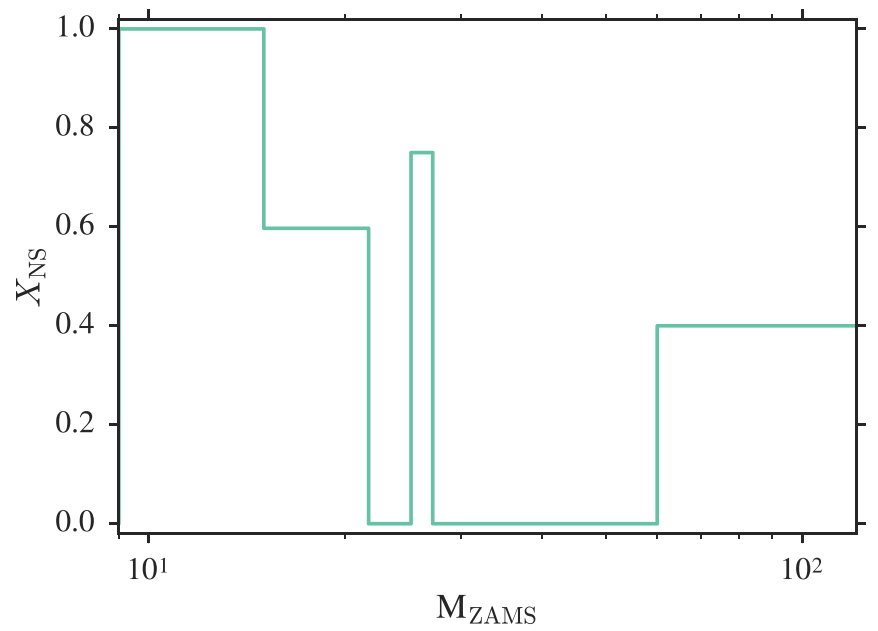

Figure 2. Fraction of neutron stars formed as a function of progenitor initial mass, for the outcomes shown in Figure 1. The initial mass has been binned to produce the minimum number of bins while still capturing whether a mass region has only neutron star outcomes, only black hole outcomes, or some combination of the two.

and $21 M_{\odot}$ and between 25 and $28 M_{\odot}$ in which the locus of pre-SN stars in this parameter space straddles the separatrix and, correspondingly, the outcome frequently changes. As a result, even very small changes in the initial conditions may turn a successful explosion into an implosion or vice versa. We therefore interpret the outcomes in these mass ranges not as rapid oscillations between the two types of remnants but rather as the co-existence of two channels with different likelihoods (see also the discussion in Clausen et al. 2015). We show this probabilistic interpretation of outcomes in Figure 2, where we plot the relative fraction of neutron stars produced, $\boldsymbol{X}_{\mathrm{NS}}$, as a function of the initial mass. We identify several regions where the outcomes can be only neutron stars, only black holes, or a combination of the two. We use these branches to appropriately weight the remnant outcomes when comparing the simulated and observed mass distributions in Sections 4 and 5.

\section{Observations of Remnant Masses}

The simulation outcomes described in Section 2 can be directly compared to the observed masses of compact objects, as we will describe in Sections 4 and 5. First, however, we review the current status of the measurements. The observed masses, which are summarized in Figure 3, come from a few primary types of observations: timing and spectra of X-ray binaries containing stellar-mass black holes, timing of millisecond pulsars with white dwarf companions as well as of the double neutron stars and, most recently, the detection of gravitational waves from black hole-black hole mergers and the first neutron star-neutron star merger.

Black hole masses are typically measured dynamically from $\mathrm{X}$-ray binaries. Data on 23 confirmed black hole X-ray binaries and on 32 additional transient systems with candidate black hole members have been compiled in Özel et al. (2010). From these data, masses are provided for 16 confirmed black holes, based on some combination of constraints on the mass ratio, inclination angle, or the mass function for the system. A similar compilation can be found in Farr et al. (2011), which focused on the masses of 15 black holes in low-mass X-ray binaries undergoing Roche-lobe overflow, as well as five black holes in wind-fed, high-mass X-ray binaries. Several masses in Figure 3

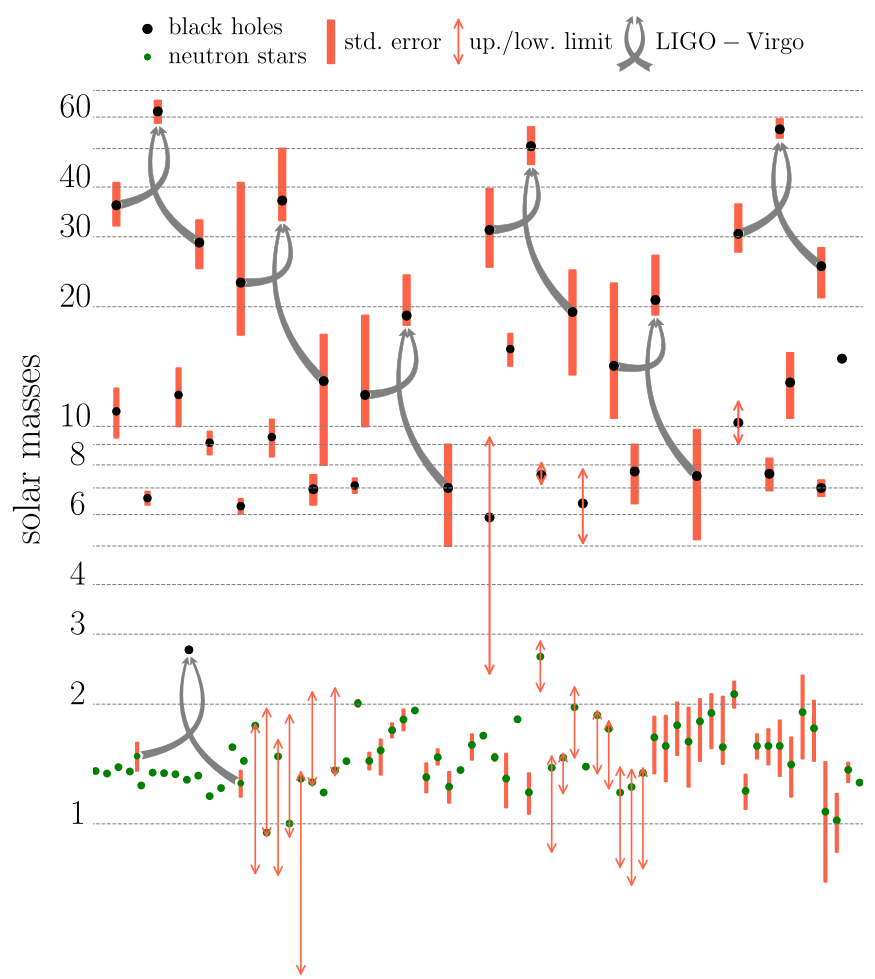

Figure 3. Observed masses of neutron stars (green points) and black holes (black points). The red vertical lines represent the error bars for each measurement. The red vertical arrows denote upper and lower measurement limits assuming a random distribution of binary inclinations, and should not be taken as Gaussian uncertainties. The gray arrows connect the progenitors to the outcome mass for each of the six confirmed and one candidate detection of gravitational waves from merging black holes and neutron stars.

are also taken from the more recent compilation found on the StellarCollapse website, ${ }^{6}$ from Wiktorowicz et al. (2014) and references therein. Finally, the most recent estimate on GX 339-4 by Heida et al. (2017) has also been included in Figure 3.

Neutron star masses are also measured dynamically, but with different methods. While spectra from an optically bright companion can be used to constrain the neutron star mass, the majority of masses are measured from radio pulsar timing. In particular, for millisecond pulsars with white dwarf companions, the measurement of any post-Keplerian parameters in the pulsar timing residuals can be used to constrain the pulsar mass, when combined with the mass function. Precision masses for 32 millisecond pulsars were recently summarized in Antoniadis et al. (2016). Precision masses for the subpopulation of double neutron stars are also determined from the timing measurement of at least two post-Keplerian parameters. For a recent review of all neutron star mass measurements, see Özel \& Freire (2016). ${ }^{7}$

Finally, there has been a new addition to these families of mass measurements, thanks to the first detections of gravitational waves by LIGO and subsequently the LIGO-Virgo Collaboration. To date, five confirmed detections have been made from the mergers of binary black holes (Abbott et al. 2016c, 2016d, 2017a; The LIGO Scientific Collaboration et al. 2017a, 2017b). The sixth set of gravitational wave black holes shown in Figure 3 is from the candidate black hole-black

\footnotetext{
6 http://www.stellarcollapse.org/bhmasses

7 The neutron star masses can be found at http://xtreme.as.arizona.edu/ NeutronStars/data/pulsar_masses.dat.
} 

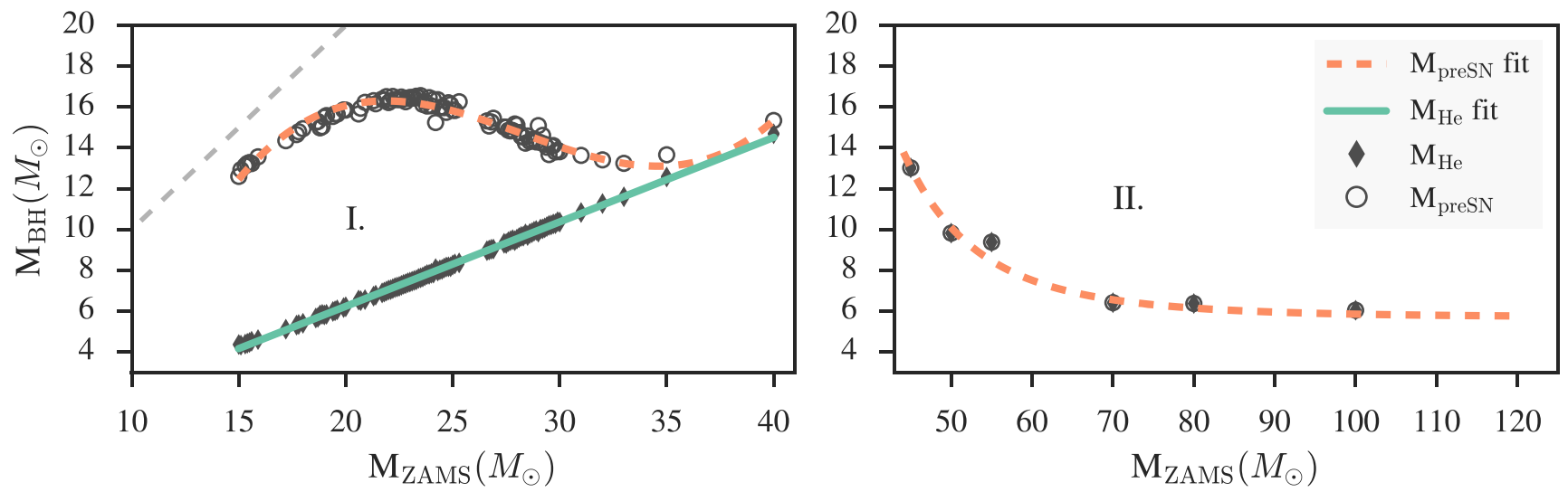

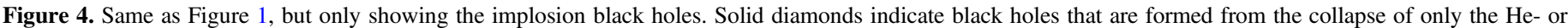

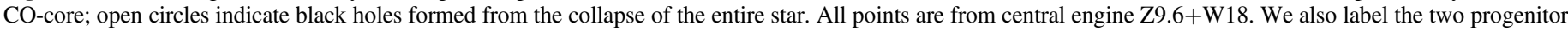

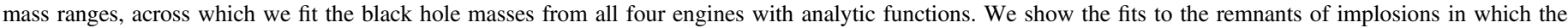
entire star collapsed and in which only the core collapsed in orange dashed and green solid lines, respectively.

hole merger LVT151012 (Abbott et al. 2016b). As shown in Figure 3, and as we will discuss more in Section 6.1, some of these black hole masses are larger than had previously been observed or even thought possible. Additionally, the first detection of gravitational waves from a neutron star-neutron star merger was recently announced (Abbott et al. 2017b), and offers a new way of adding measurements to the collection of neutron star masses as well.

The most uncertain of these masses are those that come from the measurement of a single post-Keplerian parameter in a neutron star or black hole binary, with no additional constraints on the system. Such measurements provide only an upper and lower limit to the component masses, which we represent in Figure 3 with vertical red arrows. These arrows represent a likely mass range for an assumed isotropic distribution of binary inclination and should not be interpreted as Gaussian uncertainties. We also note that Figure 3 does not include any measurements with only an upper or lower limit; we include only points with both an upper and lower limit, or with welldefined error bars. All error bars (shown as the solid red lines) represent $68 \%$ confidence intervals, except for the LIGO masses, which denote $90 \%$ confidence intervals. Finally, we note that the black hole mass measurement for NGC $300 \mathrm{X}-1$ has been excluded due to the likely asymmetric irradiation of stellar winds, which contaminates the mass measurement (T. Maccarone 2018, private communication).

\section{Black Hole Mass Distribution}

Our goal is to directly compare the outcomes of the S16 stellar evolution and SN simulations with the measured remnant masses discussed above. We first focus on the models that produce remnant masses larger than the maximum, theoretically allowed neutron star mass and, therefore, yield black holes.

The observed masses of compact objects have previously been fit with simple functional forms. The functional forms have been chosen to provide a theoretically motivated description of the data and to help facilitate a direct comparison between the observations and theory. We therefore start by creating analytic functions to model the remnant masses as a function of their ZAMS progenitor masses. By convolving these functions with the initial mass function (IMF), we determine the expected distribution, which can then be directly compared to the observed distribution.

Figure 4 shows the black hole remnant masses calculated in the simulations described in Section 2 for engines Z9.6+W18. For simplicity, we only show the outcomes from one particular engine combination in Figure 4, but, in the following analysis of black hole masses, we include results from four different engines. In S16, a total of five different engines (calibrated to SN 1987A) were applied to models with $M_{\text {ZAMS }}>12 M_{\odot}$, while only the Z9.6 engine (calibrated to SN 1054) was applied to lighter models. Out of the SN 1987A calibrated engines, engine W20 was exceptionally weak. As a result, the Z9.6 +W20 combination would have resulted in too few successful explosions to adequately explain basic nucleosynthesis and energetics constraints (Sukhbold et al. 2016). The remaining four engines gave very similar results (see Figure 13 of S16). In our analysis as well, we find that each of these four engines produce consistent results with those shown in Figure 4. Thus, for the black hole analysis, we average together the results of the four engines: W15, W18, N20, and S19.7, and use engine Z9.6 for all low-mass progenitors.

In Figure 4, we also identify two different branches of mass outcomes, so that each branch is well-approximated by a single function. The branches are divided as follows: Branch I spans $15 \leqslant M_{\text {ZAMS }} \leqslant 40 M_{\odot}$, which corresponds to the range of red supergiant models that retained significant amounts of the envelope upon implosion. Branch II spans $45 \leqslant M_{\text {ZAMS }} \leqslant 120 M_{\odot}$, which corresponds to the range of models that lost all of their envelopes and die as Wolf-Rayet stars.

In Branch I of the black hole masses, the outcomes are bounded by two possibilities: (1) He-core implosion, which occurs in the event that the entire hydrogen envelope is ejected by a weak shock during the black hole formation or has been tidally stripped by a binary companion prior to the collapse, or (2) implosion of the entire pre-SN stellar mass. In our modeling of these outcomes, we allow for a variable fraction of the envelope to be ejected, which we denote as $f_{\mathrm{ej}}$. For the more massive progenitors in Branch II, which do not retain their hydrogen and helium envelopes, $f_{\mathrm{ej}}$ has no physical meaning. For these models, the only scenario considered is the collapse of the CO-core.

Accordingly, the filled diamonds in Figure 4 indicate that the entire stellar envelope was ejected prior to or during the implosion and only the core collapsed to form the black hole. 
We represent remnant masses from this scenario as $M_{\mathrm{BH}, \mathrm{core}}$, which have an ejection fraction, $f_{\mathrm{ej}}=1$. On the other end of the spectrum, open circles indicate that the entire pre-SN star collapsed to form the black hole. We specify remnant masses from this scenario as $M_{\mathrm{BH}, \text { all }}$, with ejection fractions of $f_{\mathrm{ej}}=0$.

The gaps between these branches are due to the discrete sampling of the S16 simulations. For Branch I, we separately fit the outcomes from core-only implosions and the implosions in which the entire star collapses with simple functional forms. For Branch II, we consider only the outcomes of core-only implosions. All fits were performed with standard least-squares methods. We fit with functions that are power-law or first-, second-, or third-order polynomials, and select the optimal function by minimizing the rms of the residuals. If the rms of the residuals is $<1 \%$ for more than one of the polynomials, we take the lowest-order function. However, we note that the conclusions we find are largely independent of the particular models chosen.

The functions for $M_{\mathrm{BH} \text {,core }}$ and $M_{\mathrm{BH}, \text { all }}$ differ quite significantly from each other in Branch I because $M_{\mathrm{BH} \text {,core }}$ depends on the assumed input physics in the stellar modeling but does not depend on the mass loss for this range of progenitor masses, whereas $M_{\mathrm{BH} \text {,all }}$ is highly sensitive to the particular mass loss prescription and its efficiency. In contrast, for the stars in Branch II, the final masses of the resulting CO-cores are strongly dependent on both the assumed red supergiant and Wolf-Rayet mass loss prescriptions, but the resulting core uniquely determines the black hole mass.

In Branch I, we find that the outcomes from implosions in which only the He-core collapses $\left(f_{\mathrm{ej}}=1\right)$ are well-fit by a linear model and find

$$
\begin{aligned}
M_{\mathrm{BH}, \mathrm{core}}\left(M_{\mathrm{ZAMS}}\right)= & -2.049+0.4140 M_{\mathrm{ZAMS}}, \\
& 15 \leqslant M_{\mathrm{ZAMS}} \leqslant 40 M_{\odot} .
\end{aligned}
$$

with residuals of $0.9 \%$.

For the outcomes in Branch I for the implosions in which the entire star collapses $\left(f_{\mathrm{ej}}=0\right)$, we find that a third-order polynomial with parameters

$$
\begin{aligned}
M_{\mathrm{BH}, \mathrm{all}}\left(M_{\mathrm{ZAMS}}\right)= & 15.52-0.3294\left(M_{\mathrm{ZAMS}}-25.97\right) \\
& -0.02121\left(M_{\mathrm{ZAMS}}-25.97\right)^{2} \\
& +0.003120\left(M_{\mathrm{ZAMS}}-25.97\right)^{3} \\
& 15 \leqslant M_{\mathrm{ZAMS}} \leqslant 40 M_{\odot}
\end{aligned}
$$

is necessary to keep the residuals $\sim 1 \%$. A second-order polynomial fit produces larger residuals of $\sim 4 \%$.

In Branch II, we only consider the implosion of the CO-core, since there is no remaining envelope at these high masses. We find that fitting these outcomes with a power-law model is sufficient and find

$$
\begin{aligned}
M_{\mathrm{BH}, \mathrm{core}}\left(M_{\mathrm{ZAMS}}\right)= & 5.697+7.8598 \times 10^{8}\left(M_{\mathrm{ZAMS}}\right)^{-4.858}, \\
& 45 \leqslant M_{\mathrm{ZAMS}} \leqslant 120 M_{\odot}
\end{aligned}
$$

with residuals of $\sim 9 \%$. This larger residual is due to a single data point. Fitting instead with a second- or third-order polynomial improved the residuals by less than $\sim 0.5 \%$ and made no significant change to the final distribution, so we chose to use the simpler power-law model.

In order to probe regimes in which there may be partial ejection fractions, we can extrapolate from our fits of the special cases of $f_{\mathrm{ej}}=0$ or 1 , using the approximation

$$
\begin{aligned}
M_{\mathrm{BH}}\left(M_{\mathrm{ZAMS}} ; f_{\mathrm{ej}}\right)= & f_{\mathrm{ej}} M_{\mathrm{BH}, \mathrm{core}}\left(M_{\mathrm{ZAMS}}\right) \\
& +\left(1-f_{\mathrm{ej}}\right) M_{\mathrm{BH}, \text { all }}\left(M_{\mathrm{ZAMS}}\right) .
\end{aligned}
$$

We use this formalism for its simplicity but also note that the pre-SN core structures and the binding energy outside the He-cores are not identical in models where the stars retain some of their envelopes. As a result, the ultimate fraction of the envelope that gets ejected upon implosion is likely not the same for all progenitor masses. Considering the uncertain nature of this mechanism, in this work we adopt a simple scenario where $f_{\text {ej }}$ has the same value for all applicable stars, in order to explore its effect on the resulting black hole mass distribution.

Using this combined model for each branch, we calculate the probability of a particular black hole remnant mass as

$$
\begin{aligned}
& P\left(M_{\mathrm{BH}} \mid M_{\mathrm{ZAMS}} ; f_{\mathrm{ej}}\right) \\
& =\left|\frac{d M_{\mathrm{BH}}\left(M_{\mathrm{ZAMS}} ; f_{\mathrm{ej}}\right)}{d M_{\mathrm{ZAMS}}}\right|^{-1} \delta\left[M_{\mathrm{ZAMS}}-M_{\mathrm{ZAMS}}\left(M_{\mathrm{BH}}\right)\right],
\end{aligned}
$$

where the $\delta$-function encapsulates the relationship between $M_{\text {ZAMS }}$ and $M_{\mathrm{BH}}$ at the value at which the probability distribution is evaluated. Finally, to get the distribution of black holes for each branch, we marginalize over the progenitor masses, i.e.,

$$
P\left(M_{\mathrm{BH}} ; f_{\mathrm{ej}}\right)=\int P\left(M_{\mathrm{BH}} \mid M_{\mathrm{ZAMS}} ; f_{\mathrm{ej}}\right) P\left(M_{\mathrm{ZAMS}}\right) d M_{\mathrm{ZAMS}},
$$

where for the probability of finding a particular mass, $M_{\text {ZAMS }}$, in the initial mass distribution, we use the Salpeter IMF,

$$
P\left(M_{\mathrm{ZAMS}}\right)=C\left(M_{\mathrm{ZAMS}}\right)^{\alpha},
$$

with $\alpha=-2.3$ and $C=0.065$ (Salpeter 1955).

We weight the probability of each branch by a value, $X_{\mathrm{BH}}$, which represents the fraction of outcomes in that branch that produced black holes. These weighting fractions, which are shown in Table 1 for each engine, reflect the fact that the explosion outcomes can form either neutron stars or black holes in certain mass ranges, as discussed in Section 2, and need to be treated probabilistically. Note that we do not include fallback cases in the number of successful black holes, but do include them in the number of possible outcomes. That is,

$$
X_{\mathrm{BH}}=N_{\mathrm{BH}} /\left(N_{\mathrm{BH}}+N_{\mathrm{NS}}+N_{\mathrm{fb}}\right) \text {, }
$$

where $N_{\mathrm{BH}}$ is the number of black holes excluding the fallback cases, $N_{\mathrm{NS}}$ is the number of neutron stars, and $N_{\mathrm{fb}}$ is the number of fallback cases. We note that $X_{\mathrm{BH}}$ is related to the values of $X_{\mathrm{NS}}$ shown in Figure 2 by $X_{\mathrm{BH}} \approx\left(1-X_{\mathrm{NS}}\right),{ }^{8}$ but the fractions here are re-calculated over the specific mass ranges of Branches I and II.

\subsection{Comparing the Simulated and Observed Black Hole Mass Distributions}

We calculate the final black hole mass distribution by summing the probability contributions for each branch, as

\footnotetext{
The full relationship between the fractions is given by $X_{\mathrm{BH}}+X_{\mathrm{NS}}+$ $X_{\text {fallback }}=1$, but we have omitted the fallback cases from our analysis, since they are few in number and do not significantly affect our final results.
} 
Table 1

Fraction of Outcomes That Yield Black Holes in Each Branch of Figure 4 for the Four Central Engine Models Included in Our Analysis

\begin{tabular}{lccccc}
\hline \hline Branch & $X_{\mathrm{BH}, \mathrm{W} 15}$ & $X_{\mathrm{BH}, \mathrm{W} 18}$ & $X_{\mathrm{BH}, \mathrm{N} 20}$ & $X_{\mathrm{BH}, \mathrm{S} 19.8}$ & $X_{\mathrm{BH}, \mathrm{Avg}}$ \\
\hline $\mathrm{I}$ & 0.686 & 0.635 & 0.500 & 0.474 & 0.574 \\
II & 0.875 & 0.750 & 0.500 & 0.500 & 0.656 \\
\hline
\end{tabular}

found in Equation (6), and weighting each contribution by the ratios, $X_{\mathrm{BH}}$, of Table 1 . We include in this analysis the results from each of the four central engines, which we average together. We show the resulting black hole mass distribution, for various ejection fractions, in Figure 5.

We find that, in general, the smaller the ejection fraction, the narrower the expected mass distribution. This is because the pre-SN mass is less sensitive to the initial mass in Branch I than the He-core mass is, as shown in Figure 4. Additionally, we find that smaller ejection fractions produce larger black holes, as expected. For an ejection fraction of 0 , the distribution is confined to $M_{\mathrm{BH}} \sim 12-16 M_{\odot}$, with sharp peaks at $\sim 13$ and $16 M_{\odot}$. In contrast, we find that an ejection fraction of 0.9 leads to a mass distribution with a soft decay, spanning from $M_{\mathrm{BH}} \sim 5-12 M_{\odot}$.

In the bottom panel of Figure 5, we show two black hole mass distributions inferred from the observed black hole masses in our Galaxy. The first distribution was calculated by Özel et al. (2010) from black hole masses measured from 16 low-mass X-ray binaries. The resulting distribution was well-fit by a decaying exponential. The second distribution was calculated similarly by Farr et al. (2011) from mass measurements of 15 black holes in low-mass X-ray binaries and five black holes in high-mass, wind-fed X-ray binaries. In this study, they fit several different models to the data and found strong evidence for an exponentially decaying profile of the form,

$$
\begin{aligned}
P\left(M_{\mathrm{BH}} ; M_{\min }, M_{0}\right)= & \frac{\exp \left(M_{\min } / M_{0}\right)}{M_{0}} \\
& \times \begin{cases}\exp \left(-M_{\mathrm{BH}} / M_{0}\right), & M_{\mathrm{BH}} \geqslant M_{\min } \\
0, & M_{\mathrm{BH}}<M_{\min }\end{cases}
\end{aligned}
$$

where $M_{\min }$ is the minimum black hole mass, which was found to be $5.3268 M_{\odot}$, and $M_{0}$ is a scale mass, found to be $4.70034 M_{\odot}$ (Farr et al. 2011).

We note that the substructure in the simulated distributions is finer than the typical uncertainties in the observations and would not be observable as is. Thus, we also calculate the distribution that would be inferred from the underlying simulated distribution, by fitting our full distribution with the exponential function of Equation (9) with a standard leastsquares fitting algorithm. This "inferred" simulated distribution is shown for $f_{\mathrm{ej}}=0.9$ in the bottom panel of Figure 5, and shows close agreement with the observed distribution in our Galaxy. We report the best-fit parameters for $f_{\mathrm{ej}}=0.9$ in Table 2, along with those found in Farr et al. (2011). We find that the parameters are indeed comparable.

Finally, we calculate the likelihood of the "inferred" distributions for each ejection fraction, assuming the same
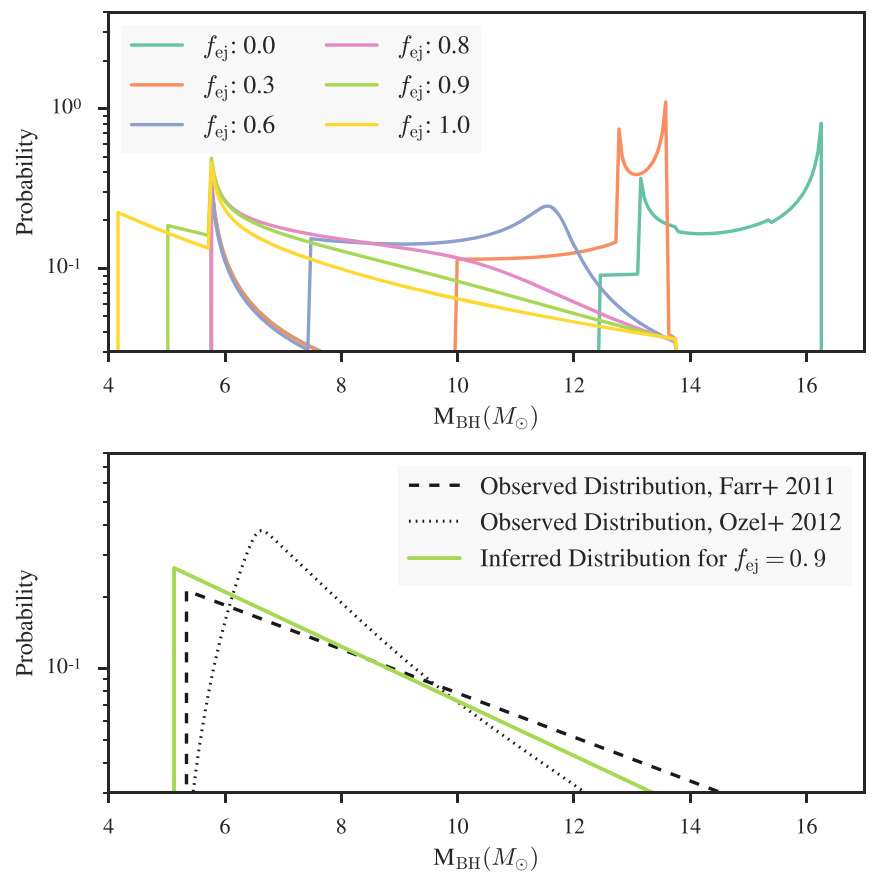

Figure 5. Top: mass distribution of black holes averaged from the simulations for the four central engines. Different colors represent different fractions of the stellar envelope that are ejected either during the implosion by a weak shock, or prior to the implosion via tidal stripping by a binary companion. Bottom: comparison to the observed mass distributions of Farr et al. (2011) and Özel et al. (2012), shown in the black dashed and dotted lines, respectively. Here, the green line represents the distribution that would be inferred from the underlying simulated distribution for $f_{\text {ej }}=0.9$, if a decaying exponential form is assumed. We find that to recreate the observed distribution, a relatively large ejection fraction is required.

Table 2

Fit Parameters for the Black Hole Mass Distribution for $f_{\mathrm{ej}}=0.9$

\begin{tabular}{lcc}
\hline \hline Source of distribution & $M_{\min }\left(M_{\odot}\right)$ & $M_{0}\left(M_{\odot}\right)$ \\
\hline Farr et al. (2011) & 5.3 & 4.7 \\
Simulated distribution & 5.1 & 3.8 \\
\hline
\end{tabular}

functional form of Equation (9). We calculate the likelihood as

$$
\mathcal{L}=\exp \left[-\sum_{i} \frac{\left(P_{\text {inferred }}\left(M_{\mathrm{BH}, \mathrm{i}} ; f_{\mathrm{ej}}\right)-P_{\mathrm{obs}}\left(M_{\mathrm{BH}, \mathrm{i}}\right)\right)^{2}}{P_{\mathrm{obs}}\left(M_{\mathrm{BH}, \mathrm{i}}\right)}\right],
$$

where $P_{\mathrm{obs}}\left(M_{\mathrm{BH}, \mathrm{i}}\right)$ is the probability given in Equation (9) for the inferred parameters from Farr et al. (2011), for black holes above the minimum mass of Farr et al. (2011) in our sampling. We show these likelihoods in Figure 6, and find that ejection fractions of $f_{\mathrm{ej}} \gtrsim 0.9$ have the highest likelihoods, given the observed mass distribution. This implies not only that the S16 simulations are closely reproducing the black hole masses observed in our Galaxy, but also that a large fraction of the stellar envelope must be ejected in order to form the observed black holes. This mass ejection most likely occurs during implosion itself but may also occur beforehand, through tidal stripping from a binary companion.

Finally we note that, for all ejection fractions in the solarmetallicity models of S16, there appear to be no black holes with masses above $12-16 M_{\odot}$. This is particularly interesting in light of the recent inferences of black holes with $M \geqslant 22 M_{\odot}$ 


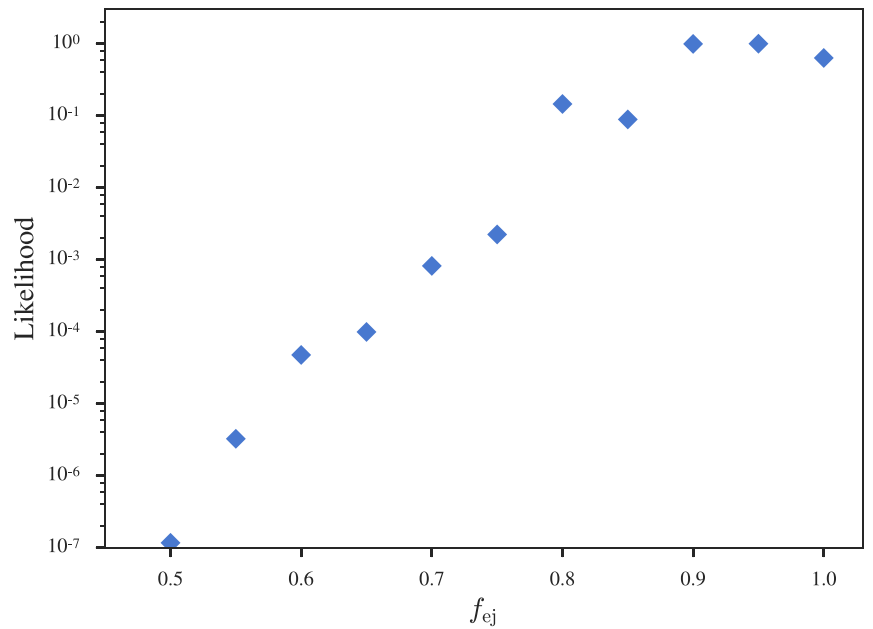

Figure 6. Likelihood that the inferred, simulated distribution matches the observed distribution of black hole masses, for various ejection fractions. We find that the likelihood peaks when a relatively large fraction of the stellar envelope has been ejected, $f_{\mathrm{ej}}>0.9$.

that have been made with the first LIGO and Virgo gravitational wave detections (Abbott et al. 2016c, 2016d, 2017a; The LIGO Scientific Collaboration et al. 2017b), as can be seen in Figure 3. We discuss this further in Section 6.1.

\section{Neutron Star Mass Distribution}

We calculate the neutron star mass distribution with the same method that we used for the black hole distribution of Section 4. Figure 7 shows the neutron star remnant masses for various progenitors, as calculated with the Z9.6+W18 engines. Even though the outcomes from engine W18 are illustrative of the outcomes for all four engines, as discussed in Section 4, averaging over the masses introduces a small level of noise that was not present in the black hole case. In order to avoid combining these slightly different sets of outcomes, we randomly choose to use the W18 engine, along with Z9.6 for the lighter models, and include only that engine combination in the neutron star analysis.

Within the neutron star remnant masses, we identify seven distinct segments that we fit with simple analytic functions, as in Section 4. We show the analytic functions that we fit to each branch in Figure 7 in purple.

We find that the first branch is best fit by a third-order polynomial with parameters

$$
\begin{aligned}
& M_{\mathrm{NS}, \mathrm{b}}\left(M_{\mathrm{ZAMS}}\right)=2.24+0.508\left(M_{\mathrm{ZAMS}}-14.75\right) \\
& +0.125\left(M_{\mathrm{ZAMS}}-14.75\right)^{2}+0.0110\left(M_{\mathrm{ZAMS}}-14.75\right)^{3}, \\
& \quad 9 \leqslant M_{\mathrm{ZAMS}} \leqslant 13 M_{\odot} .
\end{aligned}
$$

The rms of the residuals to this fit is $\sim 1.3 \%$. Here, $M_{\mathrm{NS}, \mathrm{b}}$ is the baryonic mass of the neutron star. The baryonic masses are the natural output of the stellar evolution and explosion models, which we will later convert to gravitational masses.

We find that Branches II-IV are sufficiently fit with linear models, with residuals $<1 \%$ for

$$
\begin{aligned}
M_{\mathrm{NS}, \mathrm{b}}\left(M_{\mathrm{ZAMS}}\right)= & 0.123+0.112 M_{\mathrm{ZAMS}} \\
& 13<M_{\mathrm{ZAMS}}<15 M_{\odot},
\end{aligned}
$$

$$
\begin{aligned}
M_{\mathrm{NS}, \mathrm{b}}\left(M_{\mathrm{ZAMS}}\right)= & 0.996+0.0384 M_{\mathrm{ZAMS}} \\
& 15 \leqslant M_{\mathrm{ZAMS}}<17.8 M_{\odot} .
\end{aligned}
$$

and

$$
\begin{aligned}
M_{\mathrm{NS}, \mathrm{b}}\left(M_{\mathrm{ZAMS}}\right)= & -0.020+0.10 M_{\mathrm{ZAMS}} \\
& 17.8<M_{\mathrm{ZAMS}}<18.5 M_{\odot} .
\end{aligned}
$$

We find Branch V to be approximately randomly distributed, and thus fit it with a Gaussian distribution, i.e.,

$$
P\left(M_{\mathrm{NS}, \mathrm{b}} \mid M_{\mathrm{ZAMS}}\right)=\frac{1}{\sqrt{2 \pi \sigma}} \exp \left[-\left(M_{\mathrm{NS}, \mathrm{b}}-M_{0}\right)^{2} / 2 \sigma^{2}\right],
$$

where $M_{0}$ and $\sigma$ are the mean and standard deviation of the distribution. For Branch V $\left(18.5 \leqslant M_{\text {ZAMS }}<21.7 M_{\odot}\right)$, we find the standard deviation to be $\sigma=0.158$ and the mean to be $M_{0}=1.60 M_{\odot}$.

Branch VI is best fit by a third-order polynomial with parameters

$$
\begin{aligned}
& M_{\mathrm{NS}, \mathrm{b}}\left(M_{\mathrm{ZAMS}}\right)=3232.29-409.429\left(M_{\mathrm{ZAMS}}-2.619\right) \\
& +17.2867\left(M_{\mathrm{ZAMS}}-2.619\right)^{2}-0.24315\left(M_{\mathrm{ZAMS}}-2.619\right) \\
& 25.2 \leqslant M_{\mathrm{ZAMS}}<27.5 M_{\odot},
\end{aligned}
$$

with residuals $\sim 3 \%$.

Finally, we fit Branch VII $\left(60 \leqslant M_{\text {ZAMS }} \leqslant 120 M_{\odot}\right)$ with a Gaussian distribution and find $\sigma=0.02$ and $M_{0}=1.78 M_{\odot}$. It should be noted, however, that Branch VII contains only two points; as a result, the parameters of this particular fit should be interpreted with caution. We show the Gaussian distributions on the right side of each $M_{\mathrm{ZAMS}}$ range in Figure 7.

We use the analytic functions for Branches I-IV and VI to calculate the probability distribution of neutron star masses, according to

$$
\begin{aligned}
& P\left(M_{\mathrm{NS}, \mathrm{b}} \mid M_{\mathrm{ZAMS}}\right) \\
& =\left|\frac{d M_{\mathrm{NS}, \mathrm{b}}\left(M_{\mathrm{ZAMS}}\right)}{d M_{\mathrm{ZAMS}}}\right|^{-1} \delta\left[M_{\mathrm{ZAMS}}-M_{\mathrm{ZAMS}}\left(M_{\mathrm{NS}, \mathrm{b}}\right)\right] .
\end{aligned}
$$

For Branches V and VII, we simply use the fitted Gaussian distribution for $P\left(M_{\mathrm{NS}, \mathrm{b}} \mid M_{\mathrm{ZAMS}}\right)$.

Finally, we marginalize the probabilities of each branch by

$$
P\left(M_{\mathrm{NS}, \mathrm{b}}\right)=\int P\left(M_{\mathrm{NS}, \mathrm{b}} \mid M_{\mathrm{ZAMS}}\right) P\left(M_{\mathrm{ZAMS}}\right) d M_{\mathrm{ZAMS}},
$$

and use the IMF of Equation (7) for $P$ (M $\left.M_{\mathrm{ZAMS}}\right)$, as in Section 4. We calculate the total distribution by summing the probability contributions from each branch, weighted by the fraction of outcomes that produce neutron stars $\left(X_{\mathrm{NS}}\right)$ in that branch. We list these fractions in Table 3 . We note that these values differ from those in Figure 2 because they are re-calculated over the specific mass ranges of the seven neutron star branches. We show the neutron star baryonic mass distribution, along with the contributions from each branch, in Figure 8.

We convert from the baryonic $\left(M_{\mathrm{NS}, \mathrm{b}}\right)$ to the gravitational $\left(M_{\mathrm{NS}, \mathrm{g}}\right)$ mass distribution with the transformation

$$
P\left(M_{\mathrm{NS}, \mathrm{g}}\right)=P\left(M_{\mathrm{NS}, \mathrm{b}}\right)\left|\frac{d M_{\mathrm{NS}, \mathrm{b}}}{d M_{\mathrm{NS}, \mathrm{g}}}\right|^{-1},
$$



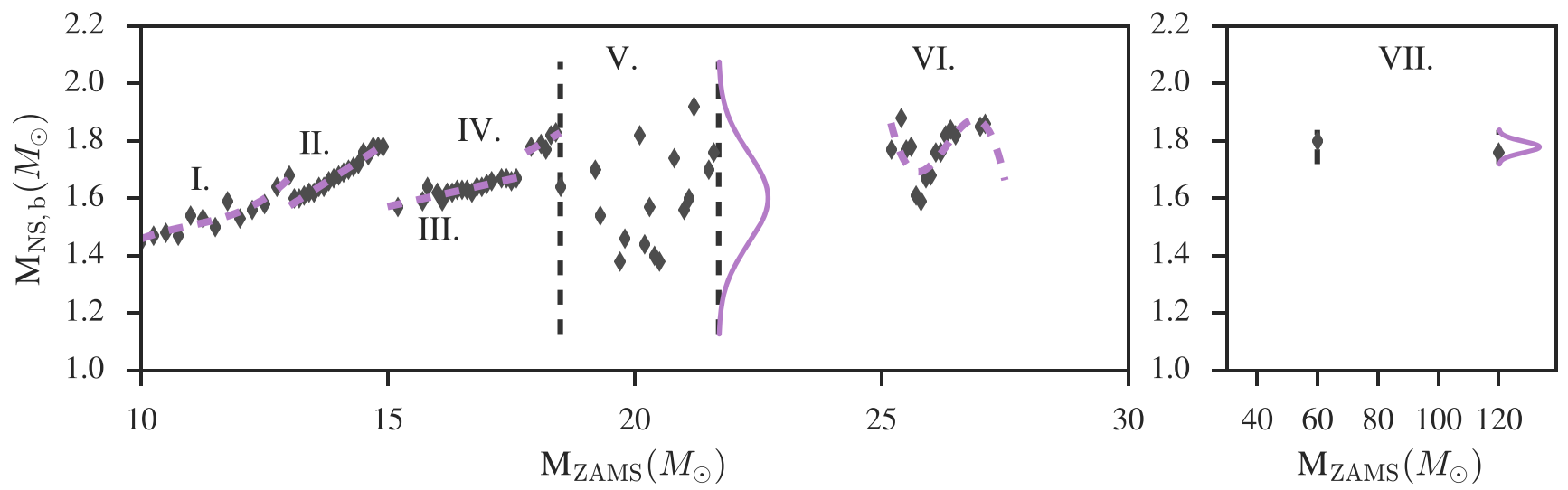

Figure 7. Neutron star baryonic masses as a function of the progenitor mass, for engines Z9.6+ W18. We identify seven distinct branches in this distribution. The analytic functions that we fit to each branch are shown in purple.

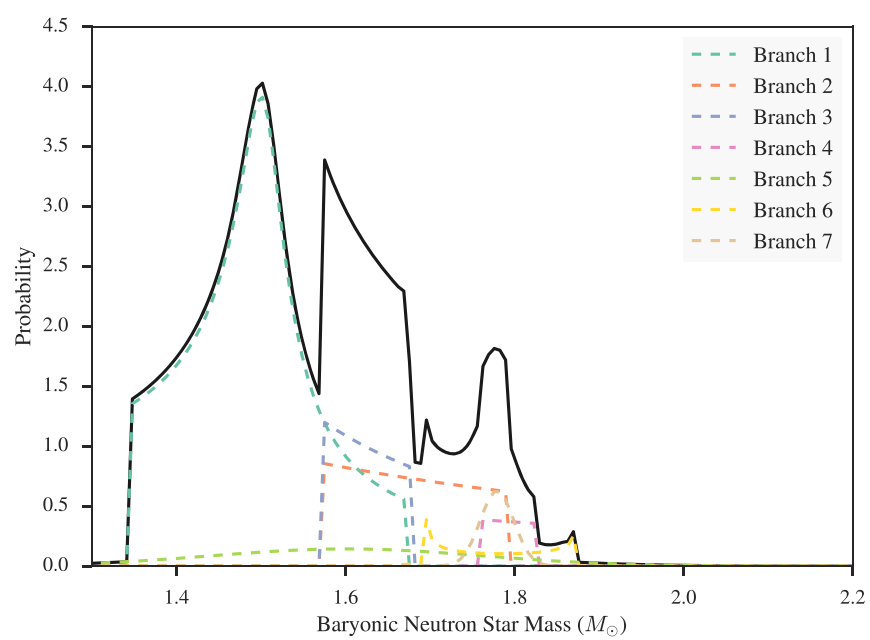

Figure 8. Baryonic mass distribution of neutron stars from the S16 simulations. The overall distribution is shown in black. The various dashed colors represent the contributions from each branch of ZAMS progenitors.

Table 3

Fraction of Outcomes That Yield Neutron Stars in Each Branch of Figure 7

\begin{tabular}{lc}
\hline \hline Branch & $X_{\text {NS, Z9.6+W18 }}$ \\
\hline I & 1.00 \\
II & 1.00 \\
III & 0.679 \\
IV & 0.833 \\
V & 0.500 \\
VI & 0.652 \\
VII & 0.400 \\
\hline
\end{tabular}

Note. Only the Z9.6+W18 engines results were included in our analysis of neutron star distributions.

where $M_{\mathrm{NS}, \mathrm{g}}$ is the gravitational mass and we calculate the derivative using the relationship between binding energy (BE) and baryonic and gravitational masses,

$$
M_{\mathrm{b}}=M_{\mathrm{g}}+\mathrm{BE} \text {. }
$$

For the BE, we use the Lattimer \& Prakash (2001) approximation

$$
\mathrm{BE}=M_{\mathrm{NS}, \mathrm{g}} \times\left(\frac{0.6 \beta}{1-0.5 \beta}\right),
$$

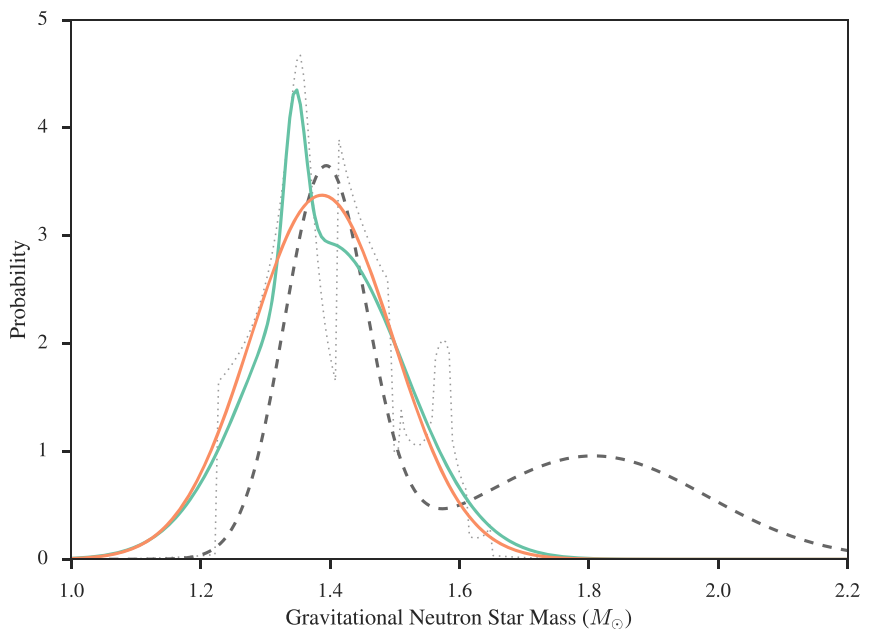

Figure 9. Gravitational mass distribution of neutron stars predicted from the S16 simulations (light gray dotted line). The bimodal distribution that would be inferred from the full simulated distribution is shown in green, while the Gaussian distribution that would be inferred from the full distribution is shown in orange. The black dashed line shows the observationally determined mass distribution of millisecond pulsars from Antoniadis et al. (2016). We find that the simulated mass distribution aligns very closely with the low-mass component of the observed distribution.

where $\beta \equiv G M_{G} / R c^{2}$ is the neutron star compactness. We find that the gravitational mass distribution depends only weakly on the choice of radius in the BE approximation, so we use a characteristic nuclear equation of state value of $12 \mathrm{~km}$. (Note, using the observationally suggested value of $11 \mathrm{~km}$ does not significantly affect the following results. For a recent review of neutron star radii, see Özel \& Freire 2016.)

\subsection{Comparing the Simulated and Observed Pulsar Mass Distributions}

We calculate the gravitational mass distribution as described in Section 5 and show the outcome as the dotted line in Figure 9. However, because the substructure between the various peaks is finer than could be detected with realistic observational uncertainties as before, we also compute and show in the same figure the single and bimodal Gaussian distributions that would be inferred from this underlying distribution, in orange and green, respectively. We fit our full distribution with a Gaussian distribution ("Model I") using 
Table 4

Fit Parameters for Neutron Star Mass Distribution in Figure 9

\begin{tabular}{|c|c|c|c|c|c|}
\hline Source of distribution & $\mu_{1}\left(M_{\odot}\right)$ & $\sigma_{1}\left(M_{\odot}\right)$ & $\mu_{2}\left(M_{\odot}\right)$ & $\sigma_{2}\left(M_{\odot}\right)$ & $r$ \\
\hline Model I-Antoniadis et al. (2016) observations & 1.542 & 0.260 & $\ldots$ & $\ldots$ & $\ldots$ \\
\hline Model I-S16 simulations & 1.387 & 0.110 & $\ldots$ & $\ldots$ & $\ldots$ \\
\hline Model II-Antoniadis et al. (2016) observations & 1.393 & 0.064 & 1.807 & 0.178 & 0.425 \\
\hline Model II-S16 simulations & 1.345 & 0.017 & 1.398 & 0.117 & 0.924 \\
\hline
\end{tabular}

Note. Model I refers to a single Gaussian model. Model II refers to the bimodal Gaussian model of Equation (22).

a standard least-squares fitting routine. We also fit our full distribution with the bimodal model ("Model II") of Antoniadis et al. (2016),

$$
P\left(M_{\mathrm{NS}} \mid \mu_{1}, \sigma_{1}, \mu_{2}, \sigma_{2}, r\right)=(1-r) G\left(\mu_{1}, \sigma_{1}\right)+r G\left(\mu_{2}, \sigma_{2}\right) \text {, }
$$

where $G(\mu, \sigma)$ are the Gaussian components and $r$ is their relative ratio. We report the most-likely fit parameters in Table 4. Finally, we also show in Figure 9 the observed neutron star mass distribution inferred from 33 millisecond pulsars in Antoniadis et al. (2016) as the black dashed line.

Model I of Antoniadis et al. (2016) is centered at $\sim 1.5 \mathrm{M}_{\odot}$. Given that the S16 simulations produce few neutron stars above 1.6 $M_{\odot}$, it is not surprising that our Gaussian fit to the simulated distribution does not agree with the Antoniadis et al. (2016) Model I, as shown in Table 4.

For the same reasons, we find no evidence of a high-mass peak when we perform the bimodal fit to the simulation outcomes. Even when exploring the full parameter space, our bimodal fit produces two peaks that are approximately aligned with the low-mass peak of the observed distribution. This may suggest that the observed low-mass peak arises from the stellar evolution models of the S16 simulations, while the high-mass peak has a separate origin. If this is true, then it may be instructive to compare the low-mass peak of the observed bimodal distribution to our single-Gaussian model. We find that these peaks agree to within their 1- $\sigma$ uncertainties. We revisit the possibility of separate origins for the two peaks in Section 6.

We can determine the origin of the low-mass peak of the observed distribution by comparing with the simulated distributions of each branch, which are shown in Figure 8. We find that progenitors in Branches I-III, i.e., with $M_{\text {ZAMS }}=9-17.8 M_{\odot}$, are the dominant contributors to the low-mass peak (contributing $\sim 86 \%$ of the total probability density) and that this peak agrees well with the observed one.

We also see that the narrow peak at $M_{\mathrm{NS}, \mathrm{g}} \sim 1.6 M_{\odot}$ originates primarily from progenitors in Branch VII, with a modest enhancement from Branch IV. Because Branch VII contains only two neutron stars that are fit with a Gaussian, its properties could easily be affected by a larger number of simulations in that mass range and it should be interpreted with caution. Finally, Branches V and VI contain very broad distributions and correspondingly do not contribute significantly to any particular peak.

In the overall comparison of neutron star masses, we find strong agreement between the simulated distribution and the low-mass peak of the observed mass distribution. We wish to emphasize here that this is a highly constrained comparison, with no parameters that can be adjusted in either distribution to improve their relative alignment. As described in Section 2, the simulations are calibrated only to reproduce the energetic properties of SN 1987A and SN 1054. The alignment that we see in Figure 9, in addition to the alignment in the black hole mass distribution, therefore, seem to be a natural consequence of calibrating to the global energetic properties. Therefore, we argue that this level of agreement in the mass distributions provides a new and independent validation of the outcomes of these stellar evolution and explosion models.

\section{Missing High-mass Remnants}

In the analyses of Section 4 and 5, we found that the remnant mass distributions predicted by the $\mathrm{S} 16$ simulations show close agreement with both the observed black hole mass distribution and low-mass neutron star distribution, offering new evidence in support of these models. However, these simulations do not produce the high-mass LIGO black holes that can be seen in Figure 3, and are missing the high-mass component of the observed pulsar mass distribution. In this section, we explore possible explanations for these discrepancies.

\subsection{High-mass Black Holes}

The recent detection of gravitational waves from black hole binary GW150914 provided the first observational evidence of "heavy" stellar-mass black holes $\left(M \gtrsim 25 M_{\odot}\right)$. The black holes in this binary were inferred to have masses of $29_{-4}^{+4}$ and $36_{-4}^{+5} M_{\odot}$ (Abbott et al. 2016d). In an initial characterization, Abbott et al. (2016a) proposed that the formation of such massive black holes via single-star evolution requires weak winds, which in turn requires an environment with metallicity $Z \lesssim 1 / 2 Z_{\odot}$. Subsequent detections have found further evidence of additional "heavy" stellar-mass black holes (Abbott et al. 2016c, 2017a; The LIGO Scientific Collaboration et al. 2017b).

The mass range of implosion outcomes due to a sample central engine applied to low metallicity progenitors is illustrated in Figure 10. The models are the ultra-low metallicity $\left(10^{-4} Z_{\odot}\right)$ "U-series" set from Sukhbold \& Woosley (2014), which consists of 110 models with initial masses between 10 and $65 M_{\odot}$. At such a low metallicity, mass loss is negligible and both the He core and final pre-SN masses increase monotonically with initial mass. At lower initial mass, the explosion landscape is similar to the solar metallicity models, since the pre-SN core structures in these stars are not strongly affected by metallicity. For more massive models, however, the cores are significantly harder to explode. Indeed, with the adopted sample central engine, all stars implode above $M_{\text {ZAMS }}>30 M_{\odot}$. From this figure, it is clear that black holes can be formed with $M_{\mathrm{BH}} \gtrsim 25 M_{\odot}$, when the metallicity is sufficiently reduced.

An alternative channel for heavy stellar-mass black hole formation from single stars has recently been proposed in the 


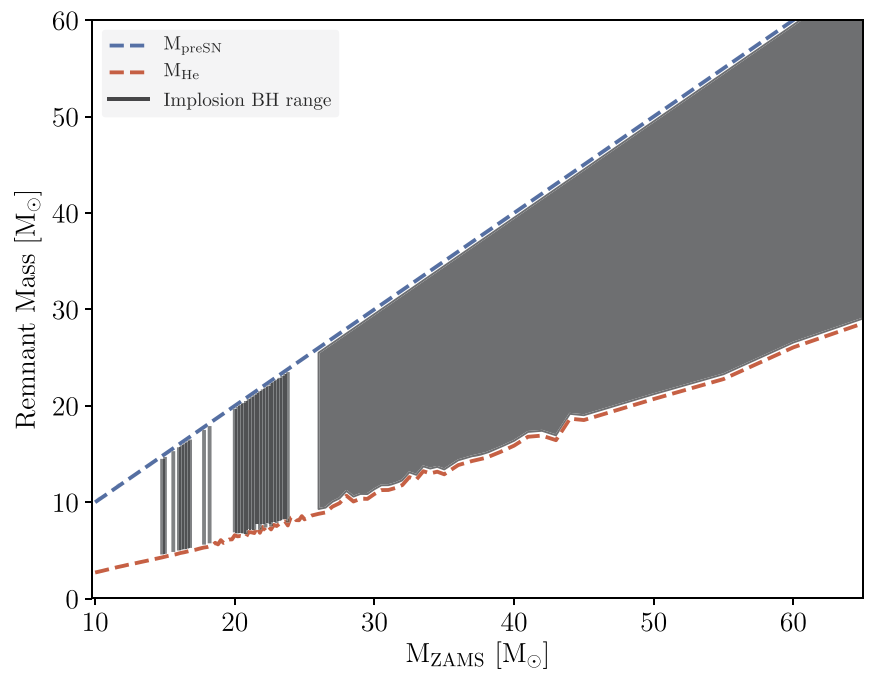

Figure 10. Range of possible black hole masses, bound by the pre-SN and $\mathrm{He}$ core masses of imploding progenitors, are shown for the ultra-low $10^{-4} Z_{\odot}$ metallicity models and a sample central engine (N20). The input physics in these models are identical to the those employed in this paper, with the exception of the reduced metallicity. Compared to the models with solar metallicity shown in Figure 4, the mass loss here is negligible and thus the implosions from the heaviest models allow the formation of heavy stellar mass black holes.

form of strong magnetic fields. Petit et al. (2017) showed that for progenitor stars with $M_{\mathrm{ZAMS}}$ in the range $40-80 M_{\odot}$, the presence of strong surface magnetic fields can significantly quench mass loss by magnetically confining a fraction of wind material to the star's surface. For a strongly magnetic $80 M_{\odot}$ star, this reduces the mass lost during the main-sequence evolution by $20 M_{\odot}$, which is the equivalent mass loss reduction achieved by reducing the stellar metallicity to $Z \sim 1 / 30 Z_{\odot}$ for a non-magnetic star (Petit et al. 2017). The S16 simulations do not include magnetic fields or rotation, but such a model offers another possible mechanism for producing black holes in the regime that was probed by the LIGO detection, without reducing the metallicity.

Numerous studies have also explored the effect of binary evolution for producing heavy stellar-mass black holes. Typically, simulations of binary massive star evolution result in the formation of a common envelope, via Roche-lobe overflow (e.g., Voss \& Tauris 2003). Accretion through the common envelope could, in principle, increase the mass of a star's He core and hence the mass of the post-SN black hole remnant to what was observed in the LIGO detections (Belczynski et al. 2016; Eldridge \& Stanway 2016; Kruckow et al. 2016; Woosley 2016; Stevenson et al. 2017). However, there remain many uncertainties in the common-envelope physics used that affect the possible outcomes (see, e.g., Ivanova et al. 2013).

It is possible to avoid the uncertainties and pitfalls of the common envelope scenario by requiring a close binary orbit. In this mechanism, the close companions tidally spin up one another. The rapid rotation then induces mixing that is faster than the chemical gradient build-up due to nuclear burning, so that the stars remain chemically homogeneous throughout hydrogen burning. This keeps the stars from developing massive hydrogen envelopes and thus offers a way in which the stars could evolve to black holes without ever undergoing significant mass transfer. It has been shown that, in such models, it is indeed possible to form heavy black holes, with
$M \gtrsim 25 M_{\odot}$ (de Mink \& Mandel 2016; Mandel \& de Mink 2016; Marchant et al. 2016). However, there remain large uncertainties in the efficiency of the mixing processes involved and in the impact of stellar winds on the orbital evolution, making it unclear whether this channel is likely or even possible (Mandel \& de Mink 2016).

Finally, many studies have found that dynamical assembly of black hole binaries in dense stellar clusters can also produce more massive black holes, via multi-body encounters, mass segregation, and gravitational focusing. These processes favor heavier black holes, which are already easier to form in the low-metallicity environments of globular clusters (Mapelli 2016; O'Leary et al. 2016; Rodriguez et al. 2016; Askar et al. 2017; Park et al. 2017). If this is the primary way in which LIGO-mass black holes are formed, the single-star evolution framework of S16 and this paper would not apply.

As a final remark, there may still be an upper bound on the expected masses of "heavy" stellar-mass black holes that might play a role in the above formation mechanisms. A recent analysis by Woosley (2017) predicts that no black holes with masses between $\sim 52$ and $133 M_{\odot}$ should be found in nature in close binary systems due to pulsational pair-instability effects. While this is in agreement with the current massive black hole detections by the LIGO-Virgo collaboration, mergers within a Hubble time from more complicated systems with more than two components could violate this bound.

\subsection{Missing High-mass Neutron Stars}

In Section 5, we also found that the high-mass peak of the observed neutron star mass distribution of Antoniadis et al. (2016) was not reproduced by the S16 simulations, despite very close agreement in the low-mass regime. Indeed, the S16 simulations do not produce any neutron stars with masses above $M_{\mathrm{NS}, \mathrm{g}}>1.7 M_{\odot}$ and it is possible that the lack of high-mass neutron stars could imply incomplete physics in the stellar evolution models. Recent work by Sukhbold et al. (2017), which employs updated physics and a denser grid of models, finds the pre-SN core structures to be intrinsically multi-valued, including for the mass range $14<M_{\mathrm{ZAMS}}<19 M_{\odot}$. Without performing full explosions of the pre-SN models, that study finds tantalizing evidence that neutron stars with $M_{\mathrm{NS}, \mathrm{g}} \sim 1.9 M_{\odot}$ can be made by the most massive stars with a significant second oxygen shell burning $\left(M_{\mathrm{ZAMS}} \sim 15 M_{\odot}\right)$. Whether these new models can recreate the high-mass peak of neutron star distribution will be explored in a future work.

Alternatively, the discrepancy would disappear if the observed high-mass peak is due to accretion rather than a second population of neutron star birth masses. However, Antoniadis et al. (2016) argue against such a path: they highlight several examples of high-mass neutron stars with companions that would be too small to allow significant accretion, inferring that the birth masses must be $\gtrsim 1.7 M_{\odot}$. Even if we allow some accretion and lower the high-mass component to $1.7 M_{\odot}$, such a population of neutron stars is still missing from the simulations. We discuss two other possibilities below.

\subsubsection{Effect of Rotation}

More massive neutron stars are generally made by more massive main sequence stars, yet only a few models with $M_{\text {ZAMS }}>30 M_{\odot}$ successfully explode in the neutrino-driven 
formalism of S16. The effects of rotation are expected to be important in the deaths of these heavier stars (e.g., Heger et al. 2005), and therefore the inclusion of rotation in the modeling of both the evolution and explosion may result in more successful explosions at higher initial mass and consequently in more massive neutron stars.

\subsection{Effect of Binary Evolution}

It is also possible that the explodability of the pre-SN stars and the resulting compact object masses are influenced by binary evolution. The potential impact of binary evolution is particularly important to consider in our comparison, since the observed black hole and pulsar masses all come from binary systems, while the models of S16 assume single-star evolution.

While the reproduction of observed compact object mass distributions in this study may suggest that binary effects are negligible, such an argument is not conclusive. As an example, single-star models had historically reproduced the observed populations of massive stars, even though binary effects were known to be important in $\sim 70 \%$ of those stars. It was eventually shown that the assumed mass loss rates had been set at least 2-3 times too high in the single-star models for most stars, and potentially up to 10 times too high for late O-type stars and early B-type stars with weak winds. These enhanced mass loss rates were effectively compensating for mass loss due to binary Roche-lobe overflow or common envelope evolution (see Smith 2014 for a review). It is natural to ask whether the single-star models of S16 may similarly include physics that is mimicking binary effects.

Given the uncertain nature of mass loss (e.g., Renzo et al. 2017), the prescriptions employed in the models of S16 may well be overestimating what is really experienced by single massive stars. In the current work, we cannot quantify to what extent the final pre-SN masses of the S16 progenitors or the ejections fractions inferred in Section 4 are influenced by binary effects. However, we can qualitatively say that the net mass loss effects from binaries would have to closely match the combined result from the employed mass loss prescriptions (Nieuwenhuijzen \& de Jager 1990; Wellstein \& Langer 1999) and the relatively high envelope ejection fraction of $f_{\mathrm{ej}} \sim 0.9$, in order to reproduce the agreement we find with the observed remnant masses.

While the uncertain mass loss rates and possible effects of binary mass transfer are taken into account in our analysis of black holes through $f_{\text {ej }}$, these effects were not explicitly accounted for for the neutron stars. The formation of a neutron star requires the successful ejection of the entire remaining stellar envelope during the SN. Accordingly, we do not expect the resulting neutron star masses to be as sensitive to pre-SN mass loss as are the black hole masses. However, it remains possible that adjusting the pre-SN mass loss rates may affect the success or failure of the explosion itself, and hence the neutron star mass distribution. These outcomes cannot be calculated a posteriori and would require different sets of calculations for both the progenitors and the explosions.

Finally, another important consideration is the assumed IMF. The formation of binary systems requires a specific set of conditions, and the formation of binaries that remain bound even after one member explodes requires an even more restrictive scenario. The mass function of binaries that can produce compact objects with bound companions, therefore, might differ from that of isolated stars. Throughout our analysis, we used the Salpeter IMF. It is possible that using a more representative mass function could increase the weighting given to the high-mass stars, and perhaps enhance a high-mass peak of neutron stars. Fully exploring such a binary mass function could be carried out using detailed population synthesis models. We note, however, that in the case of the high-mass neutron star peak, given the absence of neutron stars with masses above $1.7 M_{\odot}$ in the S16 results, modifying the IMF alone is unlikely to produce the missing high-mass peak.

\section{Conclusions}

In this paper, we directly confronted the outcomes from the stellar evolution models and neutrino-driven explosion simulations of S16 with the observed neutron star and black hole mass distributions. Given that the central engines of the simulations were calibrated only to reproduce the ${ }^{56} \mathrm{Ni}$ mass, kinetic energy, and neutrino burst timescale of SN 1987A and the kinetic energy of SN 1054, it is perhaps surprising that the remnant mass distribution from these simulations agrees so closely with the observed black hole mass distribution and the low-mass distribution neutron stars. This degree of agreement can be taken as evidence that the stellar evolution and explosion models we studied here have reached a point where they are, at least in sum, capturing the relevant stellar, nuclear, and explosion physics involved in the formation of compact objects.

In comparing the simulated and observed mass distributions, we find that the stellar evolution and explosion models are able to accurately reproduce the observed black hole distribution (Özel et al. 2010; Farr et al. 2011), if a large fraction of the stellar envelope is ejected during the $\mathrm{SN}\left(f_{\mathrm{ej}} \sim 0.9\right)$. However, the solar-metallicity models we use in this paper do not produce heavy stellar-mass black holes, the existence of which have recently been confirmed by the LIGO gravitational wave detections. We show that similar models to those used in S16 can indeed produce heavier black holes, if the metallicity is sufficiently reduced. We also review alternative mechanisms that may produce such black holes, including via rapid rotation in binary evolution or strong magnetic fields in single-star evolution, but large uncertainties remain in the current understanding of these mechanisms.

We also find very close agreement between the simulated distribution of neutron star masses and the low-mass peak of the observed bimodal distribution found by Antoniadis et al. (2016). Specifically, we find that if we fit our simulated distribution with a bimodal model, both peaks align with the low-mass peak of the observed distribution to within their 1- $\sigma$ uncertainties. From the S16 simulations, we determine that the low-mass neutron stars originate from progenitors with $M_{\text {ZAMS }} \approx 9-18 M_{\odot}$. We find that the simulations do not reproduce the observed high-mass peak at $M_{\mathrm{NS}, \mathrm{g}} \sim 1.8 M_{\odot}$. In fact, the simulated distribution ends below $M_{\mathrm{NS}, \mathrm{g}} \sim 1.7 M_{\odot}$. We explore several possibilities for this discrepancy, including that the high-mass formation channel might require consideration of the binary mass function (as opposed to the single-star IMF we use here), or consideration of additional physics, such as the impact of rotation or binary mass transfer on the explodability of high-mass progenitors.

The method we have developed here, of directly confronting the simulated outcomes with measured mass distributions, will allow further tests of new models and ultimately will allow us to better understand the formation of compact objects. With the 
framework developed here, other formation channels may be tested as well, offering a new way to constrain stellar evolution and explosion models.

We thank Thomas Ertl for his help with the simulations used in this study and for many discussions on this work. We thank Dimitrios Psaltis, Nathan Smith, and Jeremiah Murphy for helpful discussions and comments on this work. We also thank Casey Lam for input on our reported fit coefficients. Finally, we thank the anonymous referee for valuable comments that helped to improve the manuscript. C.R. is supported by the NSF Graduate Research Fellowship Program Grant DGE1143953. T.S. is partially supported by NSF grant PHY1404311 to John Beacom. F.O. gratefully acknowledges a fellowship from the John Simon Guggenheim Memorial Foundation in support of this work as well as support by the NASA grant NNX16AC56G.

\section{ORCID iDs}

Carolyn A. Raithel (i) https://orcid.org/0000-0002-1798-6668 Tuguldur Sukhbold (iD https://orcid.org/0000-0002-1728-1561

\section{References}

Abbott, B. P., Abbott, R., Abbott, T. D., et al. 2016a, ApJL, 818, L22 Abbott, B. P., Abbott, R., Abbott, T. D., et al. 2016b, PhRvX, 6, 041015 Abbott, B. P., Abbott, R., Abbott, T. D., et al. 2016c, PhRvL, 116, 241103 Abbott, B. P., Abbott, R., Abbott, T. D., et al. 2016d, PhRvL, 116, 061102 Abbott, B. P., Abbott, R., Abbott, T. D., et al. 2017a, PhRvL, 118, 221101 Abbott, B. P., Abbott, R., Abbott, T. D., et al. 2017b, PhRvL, 119, 161101 Antoniadis, J., Tauris, T. M., Ozel, F., et al. 2016, arXiv:1605.01665

Askar, A., Szkudlarek, M., Gondek-Rosińska, D., Giersz, M., \& Bulik, T. 2017, MNRAS, 464, L36

Belczynski, K., Holz, D. E., Bulik, T., \& O’Shaughnessy, R. 2016, Natur, 534,512

Belczynski, K., Wiktorowicz, G., Fryer, C. L., Holz, D. E., \& Kalogera, V. 2012, ApJ, 757, 91

Burrows, A., Hayes, J., \& Fryxell, B. A. 1995, ApJ, 450, 830

Chamel, N., Haensel, P., Zdunik, J. L., \& Fantina, A. F. 2013, IJMPE, 22 1330018

Chieffi, A., \& Limongi, M. 2013, ApJ, 764, 21

Clausen, D., Piro, A. L., \& Ott, C. D. 2015, ApJ, 799, 190

Coughlin, E. R., Quataert, E., Fernández, R., \& Kasen, D. 2017, arXiv:1710. 01746

de Mink, S. E., \& Mandel, I. 2016, MNRAS, 460, 3545

Eldridge, J. J., \& Stanway, E. R. 2016, MNRAS, 462, 3302

Ertl, T., Janka, H.-T., Woosley, S. E., Sukhbold, T., \& Ugliano, M. 2016, ApJ, 818,124

Farr, W. M., Sravan, N., Cantrell, A., et al. 2011, ApJ, 741, 103

Fernández, R., Quataert, E., Kashiyama, K., \& Coughlin, E. R. 2018, MNRAS, 476, 2366

Fryer, C. L., Belczynski, K., Wiktorowicz, G., et al. 2012, ApJ, 749, 91

Heger, A., Woosley, S. E., \& Spruit, H. C. 2005, ApJ, 626, 350
Heida, M., Jonker, P. G., Torres, M. A. P., \& Chiavassa, A. 2017, ApJ, 846, 132 Ivanova, N., Justham, S., Chen, X., et al. 2013, A\&ARv, 21, 59

Janka, H.-T., Melson, T., \& Summa, A. 2016, ARNPS, 66, 341

Janka, H.-T., \& Mueller, E. 1996, A\&A, 306, 167

Kalogera, V., \& Baym, G. 1996, ApJL, 470, L61

Kifonidis, K., Plewa, T., Janka, H.-T., \& Müller, E. 2003, A\&A, 408, 621

Kiziltan, B., Kottas, A., De Yoreo, M., \& Thorsett, S. E. 2013, ApJ, 778, 66 Kochanek, C. S. 2014, ApJ, 785, 28

Kruckow, M. U., Tauris, T. M., Langer, N., et al. 2016, A\&A, 596, A58

Lattimer, J. M., \& Prakash, M. 2001, ApJ, 550, 426

Lattimer, J. M., \& Swesty, D. F. 1991, NuPhA, 535, 331

Lovegrove, E., \& Woosley, S. E. 2013, ApJ, 769, 109

Mandel, I., \& de Mink, S. E. 2016, MNRAS, 458, 2634

Mapelli, M. 2016, MNRAS, 459, 3432

Marchant, P., Langer, N., Podsiadlowski, P., Tauris, T. M., \& Moriya, T. J. 2016, A\&A, 588, A50

Müller, B., Heger, A., Liptai, D., \& Cameron, J. B. 2016, MNRAS, 460, 742 Murphy, J. W., \& Dolence, J. C. 2017, ApJ, 834, 183

Nadezhin, D. K. 1980, Ap\&SS, 69, 115

Nieuwenhuijzen, H., \& de Jager, C. 1990, A\&A, 231, 134

Nomoto, K., Kobayashi, C., \& Tominaga, N. 2013, ARA\&A, 51, 457

O’Connor, E., \& Ott, C. D. 2011, ApJ, 730, 70

O’Leary, R. M., Meiron, Y., \& Kocsis, B. 2016, ApJL, 824, L12

Özel, F., \& Freire, P. 2016, ARA\&A, 54, 401

Özel, F., Psaltis, D., Narayan, R., \& McClintock, J. E. 2010, ApJ, 725, 1918

Özel, F., Psaltis, D., Narayan, R., \& Santos Villarreal, A. 2012, ApJ, 757, 55

Park, D., Kim, C., Lee, H. M., Bae, Y.-B., \& Belczynski, K. 2017, MNRAS, 469, 4665

Pejcha, O., \& Thompson, T. A. 2015, ApJ, 801, 90

Pejcha, O., Thompson, T. A., \& Kochanek, C. S. 2012, MNRAS, 424, 1570

Petit, V., Keszthelyi, Z., MacInnis, R., et al. 2017, MNRAS, 466, 1052

Renzo, M., Ott, C. D., Shore, S. N., \& de Mink, S. E. 2017, A\&A, 603, A118

Rhoades, C. E., \& Ruffini, R. 1974, PhRvL, 32, 324

Rodriguez, C. L., Haster, C.-J., Chatterjee, S., Kalogera, V., \& Rasio, F. A. 2016, ApJL, 824, L8

Salpeter, E. E. 1955, ApJ, 121, 161

Smith, N. 2014, ARA\&A, 52, 487

Stevenson, S., Vigna-Gómez, A., Mandel, I., et al. 2017, NatCo, 8, 14906

Sukhbold, T., Ertl, T., Woosley, S. E., Brown, J. M., \& Janka, H.-T. 2016, ApJ, 821,38

Sukhbold, T., \& Woosley, S. E. 2014, ApJ, 783, 10

Sukhbold, T., Woosley, S., \& Heger, A. 2017, arXiv:1710.03243

The LIGO Scientific Collaboration, the Virgo Collaboration, Abbott, B. P., et al. 2017a, ApJL, 851, L35

The LIGO Scientific Collaboration, the Virgo Collaboration, Abbott, B. P., et al. 2017b, PhRvL, 119, 141101

Thorsett, S. E., \& Chakrabarty, D. 1999, ApJ, 512, 288

Ugliano, M., Janka, H.-T., Marek, A., \& Arcones, A. 2012, ApJ, 757, 69

Voss, R., \& Tauris, T. M. 2003, MNRAS, 342, 1169

Weaver, T. A., Zimmerman, G. B., \& Woosley, S. E. 1978, ApJ, 225, 1021

Wellstein, S., \& Langer, N. 1999, A\&A, 350, 148

Wiktorowicz, G., Belczynski, K., \& Maccarone, T. 2014, arXiv:1312.5924

Woosley, S. E. 2016, ApJL, 824, L10

Woosley, S. E. 2017, ApJ, 836, 244

Woosley, S. E., \& Heger, A. 2007, PhR, 442, 269

Woosley, S. E., \& Heger, A. 2015, ApJ, 810, 34

Woosley, S. E., Heger, A., \& Weaver, T. A. 2002, RvMP, 74, 1015

Zhang, W., Woosley, S. E., \& Heger, A. 2008, ApJ, 679, 639 